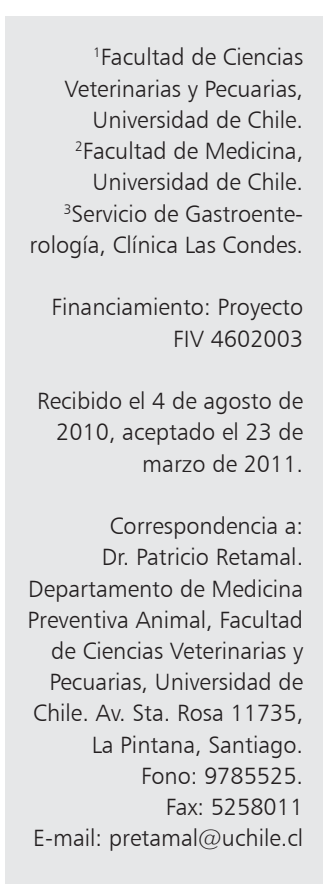

\section{Mycobacterium avium subsp paratuberculosis y enfermedad de Crohn: evidencias de una zoonosis}

\author{
PATRICIO RETAMAL M. ${ }^{1,2}$, CAROLL BELTRÁN M. ${ }^{2}$, PEDRO ABALOS P. ${ }^{1}$, \\ RODRIGO QUERA P. ${ }^{3}$, MARCELA HERMOSO R. ${ }^{2}$
}

\section{Possible association between Mycobacterium avium subsp paratuberculosis infection and Crohn's disease}

Paratuberculosis is a chronic intestinal disease of animals caused by Mycobacterium avium subsp. paratuberculosis (MAP), which has some pathological features similar to Crohn's disease (CD) in humans. The presence of MAP in food for human consumption and in affected tissues of patients with CD has been detected. Therefore, a causal association between this microorganism and the disease in humans, has been postulated. However, several related studies have failed to confirm this hypothesis and the scientific acceptance of MAP as a zoonotic agent remains controversial. This review presents the main findings related to this issue, contrasting evidences for and against an association between MAP and CD. The need to promote national studies focusing on this area is suggested.

(Rev Med Chile 2011; 139: 794-801).

Key words: Crohn disease; Mycobacterium avium; Paratuberculosis.
L a paratuberculosis bovina, también conocida como enfermedad de Johne, es patología causada por la bacteria Mycobacterium avium subsp. paratuberculosis (MAP), que se caracteriza por una lesión intestinal crónica de carácter proliferativa ${ }^{1}$. Debido a las similitudes fisiopatológicas entre la infección de los bovinos y la enfermedad de Crohn (EC) en el humano, una asociación causal del MAP con ambas patologías ha sido sugerida desde hace algunos años, lo que ha generado controversia en la comunidad científica relacionada a la transmisión inter-especies ${ }^{1,2}$. En este trabajo se revisan exhaustivamente los hallazgos más representativos y recientes que dan cuenta de esta controversia, y se describen brevemente los antecedentes en Chile que explican la necesidad de realizar estudios en este ámbito.

\section{El agente}

MAP es un patógeno intracelular facultativo, Gram positivo, ácido alcohol resistente, aerobio y cuya temperatura óptima de crecimiento es $37^{\circ} \mathrm{C}$. Con un tiempo de generación mayor a 20 h, es una bacteria de crecimiento lento que requiere varias semanas de incubación antes que sus colonias sean detectables en los medios de cultivo ${ }^{1,3,4}$. Como las bacterias del género Mycobacterium, MAP posee un genoma rico en nucleótidos $\mathrm{G}+\mathrm{C}$ y su pared celular tiene un alto contenido de ácidos micólicos. Como característica especie-específica destacan su dependencia del sideróforo micobactina para el crecimiento in vitro y la presencia del segmento de inserción IS900 en su genoma, que se ha utilizado para la detección específica de este patógeno ${ }^{1,5}$.

MAP es capaz de infectar una amplia variedad 
de especies animales, entre los que se destacan los bovinos, ovinos, caprinos, camélidos, lagomorfos, primates y carnívoros silvestres ${ }^{6,7}$. También se ha descrito infectando protozoos para sobrevivir a las condiciones medio ambientales, donde desarrolla una condición de vida intracelular dependiente de los mismos mecanismos de virulencia expresados durante la infección del macrófago, su célula blanco en la infección del hospedero animal ${ }^{8}$.

La supervivencia intracelular de MAP se debe a su capacidad de alterar la maduración fagosomal, evitar la acidificación progresiva de este compartimento y su posterior fusión con los lisosomas ${ }^{9}$, al igual que otros agentes intracelulares facultativos como M. tuberculosis, Salmonella enterica, Shigella flexneri y Brucella abortus ${ }^{10,11}$.

\section{La enfermedad en los animales}

La paratuberculosis en los animales es de distribución mundial y tiene un impacto económico considerable en la producción pecuaria ${ }^{5,12}$, debido a sus consecuencias en la disminución de la producción lechera, mayor predisposición al desarrollo de otras patologías, menor valor comercial de los animales y la eventual muerte de estos $^{5}$. La infección se transmite principalmente por contacto entre animales a través de las heces y por el consumo de calostro o leche proveniente de un animal infectado ${ }^{13}$.

La patología consiste en una enteritis crónica y linfoadenopatía mesentérica, que cursa con signos histológicos en mucosa tales como formación de granulomas dispersos que se localizan generalmente a nivel de íleon, en los linfonodos ileocecales o en la zona adyacente del colon ${ }^{1}$. En el tejido intestinal es factible observar gran cantidad de bacilos ácido alcohol resistentes a través de la tinción de Ziehl Neelsen. El análisis histopatológico demuestra la participación predominante de linfocitos, macrófagos y células plasmáticas, que pueden tener una distribución difusa o localizada dependiendo de la severidad de la infección. La inflamación granulomatosa se establece como consecuencia de la producción de citoquinas inflamatorias (tipo Th1), que llevan a la iniciación de la respuesta inmune celular del hospedero ${ }^{5,14}$.

La cronicidad de la respuesta inmune, producto de la infección por MAP, es favorecida por la alteración de mecanismos reguladores tanto locales como sistémicos, que llevan a la formación gradual de granulomas ${ }^{15}$. Debido a lo anterior, es común la ausencia de signos clínicos en el hospedero durante un prolongado período de tiempo antes de la aparición de la enfermedad ${ }^{1}$. Las lesiones granulomatosas afectan las vellosidades del epitelio mucoso a nivel del íleon, generando el síndrome de mala absorción en los animales ${ }^{1}$.

\section{La enfermedad en los humanos}

La enfermedad inflamatoria intestinal (EII) comprende un grupo de patologías crónicas cuya etiología multifactorial no está aún esclarecida del todo $^{16}$. Entre ellas destaca la EC, caracterizada por una inflamación transmural que puede involucrar segmentos de todo el tracto gastrointestinal, desde la boca al ano. El principal síntoma es la diarrea crónica e intermitente, usualmente acompañada de hemorragia, fiebre, dolor abdominal, anorexia y pérdida de peso. El trastorno de la permeabilidad intestinal, la mala absorción y la consecuente desnutrición resultan en una diversidad de manifestaciones clínicas, lo que obliga en algunos casos al uso de nutrición parenteral ${ }^{1,17,18}$. A nivel endoscópico es común observar compromiso en forma de parches, en donde áreas de mucosa afectadas son intercaladas por áreas sanas. Debido a que se trata de un daño transmural, la inflamación abarca todas las capas de la pared intestinal, comprometiendo incluso linfonodos mesentéricos. Es característico de la EC un aumento de la proliferación de las células endoteliales, debido a procesos de neoangiogénesis y linfoangiogénesis, lo que conduce a la obstrucción de vasos sanguíneos y linfáticos ${ }^{7}$. Las lesiones granulomatosas presentes en $30 \%$ de las biopsias son un signo característico, siendo su hallazgo microscópico de utilidad en el diagnóstico diferencial de otras enfermedades intestinales crónicas como la colitis ulcerosa ${ }^{1}$.

Debido al prolongado tiempo en que se desarrolla la enfermedad y al componente inflamatorio permanente que la caracteriza, se ha sugerido la participación de agentes biológicos capaces de establecer una infección crónica con una activación sostenida del sistema inmune a nivel intestinal. Como factores predisponentes en pacientes con EC se han documentado deficiencias en mecanismos de defensa antibacterianos, polimorfismos genéticos asociados a trastornos en los procesos de autofagia, la activación de componentes de autoinmunidad y la pérdida de integridad de la barrera epitelial ${ }^{19-22}$. 
Entre los posibles agentes etiológicos de EC se han descrito bacterias como MAP, Helicobacter spp, Pseudomonas spp y Yersinia spp, además de la infección con el virus Epstein-Barr y otros virus intestinales. Un factor que hace compleja la asociación causal con tales microorganismos, es la significativa modificación que presenta la microbiota comensal intestinal de pacientes con EC respecto de personas sanas, situación que puede contribuir a la severidad de la enfermedad y determinar el éxito del tratamiento ${ }^{20,23-26}$.

La hipótesis de MAP como agente causal surgió a partir de la identificación de este patógeno mediante aislamiento directo desde áreas intestinales afectadas de pacientes EC. Esta cepa clínica resultó ser patógena también para animales de laboratorio y logró reproducir la paratuberculosis en rumiantes inoculados experimentalmente ${ }^{3}$. Sin embargo, desde entonces múltiples estudios han sido realizados para comprobar esta asociación, con resultados variables en donde no siempre se ha logrado la identificación de MAP en áreas comprometidas de pacientes enfermos y a la inversa, también se ha detectado en zonas sanas ${ }^{17}$. Con estos antecedentes, la obtención de conclusiones definitivas se ha vuelto compleja y ha generado controversia respecto a la asociación de agentes biológicos y el desarrollo de esta enfermedad. A pesar de ello, diversas investigaciones tanto en el ámbito molecular como epidemiológico resultan interesantes de considerar para el esclarecimiento de estas interrogantes.

\section{La asociación MAP-EC}

A continuación se detallan los tres aspectos principales que vinculan a MAP con el cuadro de EC en humanos.

1. Desde que fueron descritas tanto la paratuberculosis bovina como la EC, se ha reportado una incidencia creciente de estos cuadros, estando actualmente ambas enfermedades en una etapa de diseminación mundial ${ }^{5,24,27}$. Además, el aumento en la incidencia de paratuberculosis en una zona geográfica determinada se ha relacionado a un aumento de la ocurrencia de EC en los años sucesivos ${ }^{27}$.

2. El desarrollo y masificación de las técnicas de biología molecular en el diagnóstico de los agentes biológicos ha incrementado la identificación de pacientes humanos con EC infectados simultáneamente por MAP. La presencia de la bacteria en estos pacientes es significativamente mayor a lo observado en personas sanas o enfermas de otras patologías intestinales ${ }^{28}$. Esto mismo se ha reportado en el cuadro de inicio temprano de la EC, donde biopsias intestinales de niños que presentan esta enfermedad tienen una incidencia significativamente mayor de MAP que niños sin $\mathrm{EC}^{24}$.

3. Pese a que varios agentes biológicos podrían estar involucrados en la etiología de EC, MAP se distingue por la generación de una enfermedad crónica mediante la inducción y mantención de una inflamación basada en la respuesta inmune celular, en un rango de distintos tipos histológicos y en diferentes hospederos, incluyendo a los primates ${ }^{25,27,29}$.

A pesar de estas evidencias, la aceptación de MAP como agente etiológico de la EC aún se encuentra en discusión. Entre los problemas que motivan la controversia, destacan el uso de métodos indirectos para la detección del agente, el escaso número de bacterias observado en los tejidos analizados, la incapacidad de algunos grupos de investigación para identificar este patógeno en pacientes con $\mathrm{EC}^{7}$ y la existencia de un amplio rango de prevalencias descritas de MAP en seres humanos, que fluctúan entre $0 \mathrm{y}$ $100 \%{ }^{30-32}$. Algunos estudios describen el empleo de diversos métodos de diagnóstico (aislamiento, histología, inmunocitoquímica y PCR), en los cuales solamente lograron detectar esta bacteria en tejidos animales utilizados como controles experimentales, pero no en biopsias de personas enfermas ${ }^{33,34}$, lo que podría reflejar diferencias en los protocolos experimentales empleados para la detección del agente. Sumado a lo anterior, las células linfoides de pacientes con EC secretan citoquinas de respuesta tanto celular como humoral frente a la estimulación con MAP, lo que sugiere un papel modificador más que causal de la bacteria. De esta manera, MAP promovería una activación inespecífica de la respuesta inmune que puede afectar las terapias con anticuerpos antiTNF utilizadas en pacientes con EC pero que no son concluyentes respecto de la asociación entre este agente y la enfermedad en las personas ${ }^{29}$. Además, el uso de antibióticos con acción sobre MAP ha resultado controversial, siendo exitoso sólo en algunos casos ${ }^{35,36}$.

Se proponen entonces algunas explicacio- 
nes que pueden dar cuenta de las discordancias observadas. Por ejemplo, la escasa cantidad de micobacterias en las lesiones analizadas se puede deber a un tipo de infección paucibacilar, caracterizada por una baja carga bacteriana en los tejidos del hospedero, o bien a la selección equívoca de muestras para aislamiento ya que serían otras áreas de tejidos donde se concentran los bacilos. Esto, porque la bacteria no sólo es capaz de infectar al macrófago sino que también infecta a las células endoteliales y a los adipocitos. Por lo tanto, en algunos casos sería más probable detectar a MAP en tejidos como los linfonódulos mesentéricos, los vasos sanguíneos, los vasos linfáticos y en los adipocitos que conforman el mesenterio ${ }^{7}$. Además, la resección quirúrgica es un procedimiento significativamente más sensible que la biopsia en el diagnóstico de MAP, sugiriendo que en humanos la bacteria podría localizarse en la capa submucosa cercana a la lesión, más que en las células epiteliales superficiales $^{37}$.

Por otra parte, se ha reportado la existencia de cepas de MAP con una pared celular reducida o inexistente, conocidas como formas L, que no se consideran ácido alcohol resistentes y por lo tanto, son indetectables mediante la tinción de Ziehl Neelsen, utilizada tradicionalmente para la observación de las bacterias del género Mycobacterium. Además, esta modificación de la pared celular de MAP disminuye su crecimiento en los medios de cultivo específicos, pasando inadvertidas cuando se ha intentado identificar en pacientes humanos ${ }^{1,27,38,39}$.

\section{Las fuentes de infección al humano}

A pesar de que MAP puede infectar diversos tejidos de los rumiantes, la leche constituye el mayor riesgo para la transmisión de la bacteria al ser humano ya sea por la contaminación de la glándula mamaria desde la sangre, por la mezcla de leches limpias e infectadas a nivel de los estanques de acopio, o bien por la contaminación de la leche con heces provenientes de animales diseminadores ${ }^{40,41}$.

De esta manera, la pasteurización corresponde al procedimiento crítico que disminuye significativamente el riesgo de transmisión de MAP a través de la leche. Sin embargo, el riesgo no se elimina totalmente ya que la estructura de la pared bacteriana, así como la capacidad de este agente de formar agrupaciones cuando se encuentra en suspensión y en alta concentración, le otorgan una mayor resistencia a procesos de descontaminación de alimentos y en particular a la pasteurización de la leche. Esto se traduce en la presencia de bacilos viables en productos lácteos comercializados al público, lo que puede constituir un factor relevante en la epidemiología de la $\mathrm{EC}^{42,43}$.

La exposición también puede darse por la contaminación de otro tipo de alimentos, ya que se ha detectado MAP en carnes bovinas, en aguas de superficie, en lagos, en la tierra, en desechos de planteles animales, en aguas servidas, etc., lo que sugiere la existencia de múltiples rutas posibles de infección para el ser humano ${ }^{7,27,44}$.

\section{El diagnóstico de MAP}

Para el diagnóstico de la infección se han desarrollado métodos para identificar el agente o bien la respuesta inmune que genera en sus hospederos. En cuanto a las técnicas que detectan la respuesta inmune humoral, estas presentan niveles variables de sensibilidad y especificidad debido a la tardía seroconversión y la reactividad cruzada con antígenos de otras micobacterias ${ }^{5,45}$. En cuanto a la detección directa del agente, ésta se realiza mediante el aislamiento bacteriológico o bien a través de la identificación del material genético. La prueba de elección para el diagnóstico sigue siendo el cultivo bacteriológico, aunque requiere un prolongado tiempo de incubación para su interpretación. Por este motivo, se ha tratado de disminuir la extensión del ensayo mediante el sistema "Micobacterial Growth Indicator Tube" (MGIT), que además ha mejorado su sensibilidad ${ }^{37}$.

Con respecto a la detección del material genético, la hibridación in situ y la prueba de PCR se han constituido en las opciones más utilizadas en los distintos tipos de muestras estudiadas, incluyendo leche, heces o tejidos, además de tejidos fijados en formalina o embebidos en parafina ${ }^{46-51}$. La secuenciación del genoma completo de MAP ha permitido la identificación y descripción de diversos marcos de lectura específicos que se constituyen en potenciales biomarcadores para el diagnóstico de la infección en los hospederos humanos y animales ${ }^{52,53}$. Desde la aplicación del PCR en los estudios de MAP, la secuencia de inserción IS900 ha sido el principal blanco de amplificación utilizado, ya que sus 14 a 18 copias en el genoma bacteriano permiten resultados de alta sensibilidad. Aunque se ha reportado la exis- 
tencia de secuencias muy similares en organismos no relacionados ${ }^{54,55}$, la IS900 sigue considerándose de una especificidad aceptable como biomarcador de MAP $^{5,19,56,57}$.

La prueba de PCR, sin embargo, presenta algunos cuestionamientos relacionados a su incapacidad de discriminar entre bacterias viables y muertas que pueden o no estar participando del proceso inflamatorio ${ }^{58}$. Por ello, se han evaluado protocolos in situ que detectan el genoma bacteriano directamente en tejidos comprometidos, otorgando evidencias sugerentes de la asociación MAP-EC ${ }^{50}$.

Como se mencionó previamente, la selección de la muestra es importante al momento de investigar la presencia de MAP en pacientes EC, debido a la distribución heterogénea y a la escases de bacterias en la forma paucibacilar de la infección ${ }^{7}$. Por lo mismo, una amplia variedad de muestras distintas se han reportado en la literatura, destacándose los tejidos intestinales, las biopsias de nódulos linfáticos, las células mononucleares de sangre periférica y la leche $e^{24,37,51,59}$. La presencia de bacterias en leche de mujeres lactantes ha sugerido que MAP también se transmite por esta vía en el ser humano, al igual que lo descrito en animales ${ }^{60}$.

\section{La situación nacional}

En Chile, la paratuberculosis en los animales es una enfermedad endémica, habiéndose reportado el aislamiento de MAP desde rebaños bovinos, caprinos y ovinos ${ }^{61-64}$ y existiendo antecedentes serológicos que sugieren más de $50 \%$ de los rebaños bovinos lecheros afectados, similar a lo descrito en Norteamérica y Europa ${ }^{27,62}$. En nuestro laboratorio se han realizado estudios durante los últimos 5 años, utilizando una prueba de Elisa (IDEXX $^{\circledR}$ Herdcheck) para detectar respuesta humoral en un total de 775 muestras de bovinos, 869 de caprinos y 3129 de ovinos, obteniéndose tasas de reacción de $13,4 \%, 9,8 \%$ y $3,1 \%$ respectivamente. Esto sugiere una amplia diseminación del agente en el ganado criado entre las regiones de Valparaíso y del Maule, comprendidas en estos análisis.

En relación a la EC en Chile, un estudio previo documentó que de un total de 282 pacientes con EII atendidos entre 1990 y 2002, 181 de ellos tenían colitis ulcerosa $(70,15 \%)$ mientras que 57 presentaban EC (20,1\%). Sin embargo, casi 70\% de los pacientes con EC fueron diagnosticados en la segunda mitad del período en estudio (19962002), sugiriendo un incremento en la incidencia de la enfermedad ${ }^{65}$.

Hasta hoy, no existe ninguna publicación nacional en que se haya intentado establecer la presencia de MAP en los tejidos de personas con EC.

\section{Conclusiones}

MAP es el agente causal de la paratuberculosis en animales, existiendo diversas evidencias que sugieren su capacidad de infectar al humano y participar en el desarrollo de la EC, enfermedad con similitudes fisiopatológicas a la paratuberculosis de los animales. MAP está presente en el medio ambiente y puede contaminar diversos tipos de alimentos por los cuales transmitirse al ser humano y los animales. El aislamiento bacteriológico y la identificación molecular del agente en el tejido intestinal humano desde un porcentaje importante de pacientes EC, dan cuenta de la asociación MAP-EC. Sin embargo, la causalidad de esta asociación es controversial debido a la existencia de estudios que no han logrado detectar MAP en personas enfermas, y porque el papel específico que tendría la bacteria en el desarrollo de la patología aún es desconocido. Si la presencia de MAP es la causa o una consecuencia de la pérdida de integridad epitelial en la EC, son interrogantes que aún deben ser esclarecidas. En Chile no se han desarrollado trabajos dirigidos a dilucidar la asociación MAP-EC, siendo de gran necesidad y relevancia el establecimiento de una red de investigadores que colaboren y permitan abordar este problema.

\section{Referencias}

1. Chacón O, Bermúdez LE, Barletta RG. Johne's disease, inflammatory bowel disease, and Mycobacterium paratuberculosis. Annu Rev Microbiol 2004; 58: 329-63.

2. Chamberlin W, Graham DY, Hulten K, El-Zimaity HM, Schwartz MR, Naser S, et al. Review article: Mycobacterium avium subsp. paratuberculosis as one cause of Crohn's disease. Aliment Pharmacol Ther 2001; 15 (3): $337-46$.

3. Chiodini RJ, Van Kruiningen HJ, Thayer WR, Merkal RS, Coutu JA. Possible role of mycobacteria in inflammatory bowel disease. I. An unclassified Mycobacterium species 
isolated from patients with Crohn's disease. Dig Dis Sci 1984; 29 (12): 1073-9.

4. Stehman SM. Paratuberculosis in small ruminants, deer, and South American camelids. Vet Clin North Am Food Anim Pract 1996; 12 (2): 441-55.

5. Harris NB, Barletta RG. Mycobacterium avium subsp. paratuberculosis in Veterinary Medicine. Clin Microbiol Rev 2001; 14 (3): 489-512.

6. Manning EJ, Collins MT. Mycobacterium avium subsp. paratuberculosis: pathogen, pathogenesis and diagnosis. Rev Sci Tech 2001; 20 (1): 133-50.

7. Pierce ES. Where are all the Mycobacterium avium subspecies paratuberculosis in patients with Crohn's disease? PLoS Pathog 2009; 5 (3): e1000234.

8. Danelishvili L, Wu M, Stang B, Harriff M, Cirillo SL, Cirillo JD, et al. Identification of Mycobacterium avium pathogenicity island important for macrophage and amoeba infection. Proc Natl Acad Sci U S A 2007; 104 (26): 11038-43.

9. Kuehnel MP, Goethe R, Habermann A, Mueller E, Rohde M, Griffiths G, et al. Characterization of the intracellular survival of Mycobacterium avium ssp. paratuberculosis: phagosomal $\mathrm{pH}$ and fusogenicity in J774 macrophages compared with other mycobacteria. Cell Microbiol 2001; 3 (8): 551-66.

10. Cossart P, Sansonetti PJ. Bacterial invasion: the paradigms of enteroinvasive pathogens. Science 2004; 304 (5668): 242-8.

11. Diacovich L, Gorvel JP. Bacterial manipulation of innate immunity to promote infection. Nat Rev Microbiol 2010; 8 (2): 117-28.

12. Judge J, Kyriazakis I, Greig A, Davidson RS, Hutchings MR. Routes of intraspecies transmission of Mycobacterium avium subsp. paratuberculosis in rabbits (Oryctolagus cuniculus): a field study. Appl Environ Microbiol 2006; 72 (1): 398-403.

13. Nielsen SS, Bjerre H, Toft N. Colostrum and milk as risk factors for infection with Mycobacterium avium subspecies paratuberculosis in dairy cattle. J Dairy Sci 2008; 91 (12): 4610-5.

14. Valentin-Weigand P, Goethe R. Pathogenesis of Mycobacterium avium subspecies paratuberculosis infections in ruminants: still more questions than answers. Microbes Infect 1999; 1 (13): 1121-7.

15. Woo SR, Czuprynski CJ. Tactics of Mycobacterium avium subsp. paratuberculosis for intracellular survival in mononuclear phagocytes. J Vet Sci 2008; 9 (1): 1-8.

16. Sepúlveda SE, Beltrán CJ, Peralta A, Rivas P, Rojas N, Figueroa C, et al. [Inflammatory bowel diseases: an immunological approach]. Rev Med Chile 2008; 136 (3): 367-75.
17. Grant IR. Zoonotic potential of Mycobacterium avium ssp. paratuberculosis: the current position. J Appl Microbiol 2005; 98 (6): 1282-93.

18. Korzenik JR. Past and current theories of etiology of IBD: toothpaste, worms, and refrigerators. J Clin Gastroenterol 2005; 39 (4 Suppl 2): S59-65.

19. Autschbach F, Eisold S, Hinz U, Zinser S, Linnebacher M, Giese T, et al. High prevalence of Mycobacterium avium subspecies paratuberculosis IS900 DNA in gut tissues from individuals with Crohn's disease. Gut 2005; 54 (7): 944-9.

20. Lowe AM, Yansouni CP, Behr MA. Causality and gastrointestinal infections: Koch, Hill, and Crohn's. Lancet Infect Dis 2008; 8 (11): 720-6.

21. Klionsky DJ. Crohn's disease, autophagy, and the Paneth cell. N Engl J Med 2009; 360 (17): 1785-6.

22. Deretic V. Links between autophagy, innate immunity, inflammation and Crohn's disease. Dig Dis 2009; 27 (3): 246-51.

23. Frank DN, St Amand AL, Feldman RA, Boedeker EC, Harpaz N, Pace NR. Molecular-phylogenetic characterization of microbial community imbalances in human inflammatory bowel diseases. Proc Natl Acad Sci U S A 2007; 104 (34): 13780-5.

24. Kirkwood CD, Wagner J, Boniface K, Vaughan J, Michalski WP, Catto-Smith AG, et al. Mycobacterium avium subspecies paratuberculosis in children with early-onset Crohn's disease. Inflamm Bowel Dis 2009; 15 (11): 1643 55.

25. Sutton CL, Kim J, Yamane A, Dalwadi H, Wei B, Landers $C$, et al. Identification of a novel bacterial sequence associated with Crohn's disease. Gastroenterology 2000; 119 (1): 23-31.

26. Laharie D, Asencio C, Asselineau J, Bulois P, Bourreille A, Moreau J, et al. Association between entero-hepatic Helicobacter species and Crohn's disease: a prospective cross-sectional study. Aliment Pharmacol Ther 2009; 30 (3): 283-93.

27. Hermon-Taylor J. Mycobacterium avium subspecies paratuberculosis, Crohn's disease and the Doomsday scenario. Gut Pathog 2009; 1 (1): 15-21.

28. Sechi LA, Scanu AM, Molicotti P, Cannas S, Mura M, Dettori G, et al. Detection and Isolation of Mycobacterium avium subspecies paratuberculosis from intestinal mucosal biopsies of patients with and without Crohn's disease in Sardinia. Am J Gastroenterol 2005; 100 (7): 1529-36.

29. Sibartie S, Scully P, Keohane J, O’Neill S, O’Mahony J, O'Hanlon D, et al. Mycobacterium avium subsp. paratuberculosis (MAP) as a modifying factor in Crohn's disease. Inflamm Bowel Dis 2010; 16 (2): 296-304. 
30. Quirke P. Antagonist Mycobacterium avium subspecies paratuberculosis is a cause of Crohn's disease. Gut 2001; 49 (6): 757-60.

31. Feller M, Huwiler K, Stephan R, Altpeter E, Shang A, Furrer $\mathrm{H}$, et al. Mycobacterium avium subspecies paratuberculosis and Crohn's disease: a systematic review and meta-analysis. Lancet Infect Dis 2007; 7 (9): 607-13.

32. Sartor RB. Does Mycobacterium avium subspecies paratuberculosis cause Crohn's disease? Gut 2005; 54 (7): 896-8.

33. Parrish NM, Radcliff RP, Brey BJ, Anderson JL, Clark DL, Jr., Koziczkowski JJ, et al. Absence of Mycobacterium avium subsp. paratuberculosis in Crohn's patients. Inflamm Bowel Dis 2009; 15 (4): 558-65.

34. Ellingson JL, Cheville JC, Brees D, Miller JM, Cheville NF. Absence of Mycobacterium avium subspecies paratuberculosis components from Crohn's disease intestinal biopsy tissues. Clin Med Res 2003; 1 (3): 217-26.

35. Selby W, Pavli P, Crotty B, Florin T, Radford-Smith G, Gibson P, et al. Two-year combination antibiotic therapy with clarithromycin, rifabutin, and clofazimine for Crohn's disease. Gastroenterology 2007; 132 (7): 2313-9.

36. Hermon-Taylor J. Treatment with drugs active against Mycobacterium avium subspecies paratuberculosis can heal Crohn's disease: more evidence for a neglected public health tragedy. Dig Liver Dis 2002; 34 (1): 9-12.

37. Schwartz D, Shafran I, Romero C, Piromalli C, Biggerstaff J, Naser N, et al. Use of short-term culture for identification of Mycobacterium avium subsp. paratuberculosis in tissue from Crohn's disease patients. Clin Microbiol Infect 2000; 6 (6): 303-7.

38. Sechi LA, Mura M, Tanda F, Lissia A, Solinas A, Fadda $\mathrm{G}$, et al. Identification of Mycobacterium avium subsp. paratuberculosis in biopsy specimens from patients with Crohn's disease identified by in situ hybridization. J Clin Microbiol 2001; 39 (12): 4514-7.

39. Onwuamaegbu ME, Belcher RA, Soare C. Cell walldeficient bacteria as a cause of infections: a review of the clinical significance. J Int Med Res 2005; 33 (1): 1-20.

40. Grant IR, Ball HJ, Rowe MT. Incidence of Mycobacterium paratuberculosis in bulk raw and commercially pasteurized cows' milk from approved dairy processing establishments in the United Kingdom. Appl Environ Microbiol 2002; 68 (5): 2428-35.

41. Grant IR. Mycobacterium paratuberculosis and milk. Acta Vet Scand 2003; 44 (3-4): 261-6.

42. Grant IR, Williams AG, Rowe MT, Muir DD. Efficacy of various pasteurization time-temperature conditions in combination with homogenization on inactivation of Mycobacterium avium subsp. paratuberculosis in milk. Appl Environ Microbiol 2005; 71 (6): 2853-61.
43. Eltholth MM, Marsh VR, Van Winden S, Guitian FJ. Contamination of food products with Mycobacterium avium paratuberculosis: a systematic review. J Appl Microbiol 2009; 107 (4): 1061-71.

44. Pierce ES. Possible transmission of Mycobacterium avium subspecies paratuberculosis through potable water: lessons from an urban cluster of Crohn's disease. Gut Pathog 2009; 1 (1): 17-21.

45. Nielsen SS, Toft N. Age-specific characteristics of ELISA and fecal culture for purpose-specific testing for paratuberculosis. J Dairy Sci 2006; 89 (2): 569-79.

46. Plante Y, Remenda BW, Chelack BJ, Haines DM. Detection of Mycobacterium paratuberculosis in formalin-fixed paraffin-embedded tissues by the polymerase chain reaction. Can J Vet Res 1996; 60 (2): 115-20.

47. Whittington RJ, Reddacliff L, Marsh I, Saunders V. Detection of Mycobacterium avium subsp. paratuberculosis in formalin-fixed paraffin-embedded intestinal tissue by IS900 polymerase chain reaction. Aust Vet J 1999; 77 (6): 392-7.

48. OIE. Manual of Diagnostic Tests and Vaccines for Terrestrial Animals: World Organisation for Animal Health 2009; 2: 276-91.

49. Grant IR, Pope CM, O’Riordan LM, Ball HJ, Rowe MT. Improved detection of Mycobacterium avium subsp. paratuberculosis in milk by immunomagnetic PCR. Vet Microbiol 2000; 77 (3-4): 369-78.

50. Jeyanathan M, Alexander DC, Turenne CY, Girard C, Behr MA. Evaluation of in situ methods used to detect Mycobacterium avium subsp. paratuberculosis in samples from patients with Crohn's disease. J Clin Microbiol 2006; 44 (8): 2942-50.

51. Hulten K, El-Zimaity HM, Karttunen TJ, Almashhrawi A, Schwartz MR, Graham DY, et al. Detection of Mycobacterium avium subspecies paratuberculosis in Crohn's diseased tissues by in situ hybridization. Am J Gastroenterol 2001; 96 (5): 1529-35.

52. Li L, Bannantine JP, Zhang Q, Amonsin A, May BJ, Alt D, et al. The complete genome sequence of Mycobacterium avium subspecies paratuberculosis. Proc Natl Acad Sci U S A 2005; 102 (35): 12344-9.

53. Semret M, Alexander DC, Turenne CY, de Haas P, Overduin $\mathrm{P}$, van Soolingen D, et al. Genomic polymorphisms for Mycobacterium avium subsp. paratuberculosis diagnostics. J Clin Microbiol 2005; 43 (8): 3704-12.

54. Englund S, Bolske G, Johansson KE. An IS900-like sequence found in a Mycobacterium sp. other than Mycobacterium avium subsp. paratuberculosis. FEMS Microbiol Lett 2002; 209 (2): 267-71.

55. Stabel JR, Bannantine JP. Development of a nested PCR method targeting a unique multicopy element, ISMap02, 
for detection of Mycobacterium avium subsp. paratuberculosis in fecal samples. J Clin Microbiol 2005; 43 (9): 4744-50.

56. Herthnek D, Nielsen SS, Lindberg A, Bolske G. A robust method for bacterial lysis and DNA purification to be used with real-time PCR for detection of Mycobacterium avium subsp. paratuberculosis in milk. J Microbiol Methods 2008; 75 (2): 335-40.

57. Khare S, Ficht TA, Santos RL, Romano J, Ficht AR, Zhang S, et al. Rapid and sensitive detection of Mycobacterium avium subsp. paratuberculosis in bovine milk and feces by a combination of immunomagnetic bead separation-conventional PCR and real-time PCR. J Clin Microbiol 2004; 42 (3): 1075-81.

58. Chamberlin WM, Naser SA. Integrating theories of the etiology of Crohn's disease. On the etiology of Crohn's disease: questioning the hypotheses. Med Sci Monit 2006; 12 (2): RA27-33.

59. Naser SA, Ghobrial G, Romero C, Valentine JF. Culture of Mycobacterium avium subspecies paratuberculosis from the blood of patients with Crohn's disease. Lancet 2004; 364 (9439): 1039-44.

60. Naser SA, Schwartz D, Shafran I. Isolation of Mycobac- terium avium subsp paratuberculosis from breast milk of Crohn's disease patients. Am J Gastroenterol 2000; 95 (4): 1094-5.

61. Soto J, Kruze J, Leiva S. Aislamiento de Mycobacterium avium subsp. paratuberculosis de fecas en rebaños lecheros infectados mediante el método de Cornell modificado. Arch Med Vet 2002; 34 (2): 275-282.

62. van Schaik G, Haro F, Mella A, Kruze J. Bayesian analysis to validate a commercial ELISA to detect paratuberculosis in dairy herds of southern Chile. Prev Vet Med 2007; 79 (1): 59-69.

63. Kruze J, Salgado M, Paredes E, Mella A, Collins MT. Goat paratuberculosis in Chile: first isolation and confirmation of Mycobacterium avium subspecies paratuberculosis infection in a dairy goat. J Vet Diagn Invest 2006; 18 (5): 476-9.

64. Salgado M, Kruze J, Collins MT. Diagnosis of paratuberculosis by fecal culture and ELISA on milk and serum samples in two types of Chilean dairy goat herds. J Vet Diagn Invest 2007; 19 (1): 99-102.

65. Figueroa C, Quera R, Valenzuela J, Jensen C. Enfermedades inflamatorias intestinales: Experiencia de dos centros chilenos. Rev Med Chile 2005; 133: 1295-304. 